\title{
Generalized Projective Synchronization Based on Active Control Method
}

\author{
Yuchun Bi ${ }^{*}$, Xiaofeng Wang and Lianyu Chen \\ Department of Electrical Engineering, Jiangsu University of Technology, Changzhou, Jiangsu, 213001, China
}

\begin{abstract}
Based on active control method, this paper successfully realizes generalized projective synchronization for robust chaotic systems with exponential item that Bao Bocheng etc. proposed. The numerical simulation results verify validity of generalized projective synchronization controller. Meanwhile, the design of the controller circuit and its simulation results also show the circuit realization's feasibility and that the design of generalized projective synchronization controller is correct.
\end{abstract}

Keywords: Active control method, generalized projective synchronization, numerical and circuit simulation.

\section{INTRODUCTION}

Bao Bocheng et al. proposed a three-dimensional robust chaos system with exponential item [1], and realized with circuit [2]. This article is a study on the applicability of synchronization control of three-dimensional chaotic system, to achieve generalized projective synchronization for two chaotic systems using active control method. The generalized projective synchronization studied in this paper refers to link the generalized synchronization and projective synchronization together, and to achieve a chaotic signal having arbitrary ratio to the output of original driving chaotic system by a scale factor [3-5]. And the correctness of the method is verified through the theoretical analysis and numerical and circuit simulation.

\section{ROBUST CHAOS SYSTEM WITH EXPONENTIAL}

Bao Bocheng et al. [1] proposed a three-dimensional continuous autonomous dissipative chaotic system, the system equations contain 5 items on the right with the absence of constant controller, as a least item for three-dimensional system that generate chaotic stream, the mathematical model is as follows:

$$
\left\{\begin{array}{l}
\dot{x}=y-x \\
\dot{y}=x z+c \\
\dot{z}=\mu-\exp (x y)
\end{array}\right.
$$

Where $\mu$ is a real constant, $\mathrm{c}$ is a constant control parameters, $\mathrm{x}, \mathrm{y}$ and $\mathrm{z}$ are state variables. The new system on the right equation contains an index square nonlinear term, a square nonlinear term, a constant parameter and two linear terms. When $\mu=5$ and $\mathrm{c}=0$, the system (1) generates a typical double scroll chaos, as shown in Fig. (1a and 1b); when $\mu=5$ and $c=2$, the system (1) generates a typical single

*Address correspondence to this author at the Department of Electrical Engineering, Jiangsu University of Technology, Changzhou, Jiangsu, 213001, China; Tel: 086-519-86953020; Fax: 086-519-86953016; E-mail: biyuchun@jsut.edu.cn scroll chaotic attractor, as shown in Fig. (1c). Literature illustrated the dynamic behavior of the chaotic system specifically, and the results illustrate that the positive Lyapunov exponent area for system (1) is wide within parameter $\mu$ change range, which means the chaotic characteristics of the system are robust.

\section{DESIGN OF GENERALIZED PROJECTIVE SYN- CHRONIZATION CONTROLLER}

The drive and response vectors achieve projective synchronization by scaling factor $k$, which also means the vector is adjustable. The system (1) is used as a driving system, which modified as is follows:

$$
\left\{\begin{array}{l}
\dot{x}=y_{1}-x_{1} \\
\dot{y}_{1}=x_{1} z_{1}+c \\
\dot{z}_{1}=\mu-\exp \left(x_{1} y_{1}\right)
\end{array}\right.
$$

The controlled response system:

$$
\left\{\begin{array}{l}
\dot{x}_{2}=y_{2}-x_{2}+u_{1} \\
\dot{y}_{2}=x_{2} z_{2}+c+u_{2} \\
\dot{z}_{2}=\mu-\exp \left(x_{2} y_{2}\right)+u_{3}
\end{array}\right.
$$

Where $u_{1}, u_{2}, u_{3}$ are synchronous controllers.

Suppose error signals $e_{1}=x_{1}-k x_{2}, e_{2}=y_{1}-k y_{2}, e_{3}=z_{1}-$ $k z_{2}$, where the scaling factor is constant $k$, error equation as follows:

$$
\left\{\begin{array}{l}
\dot{e}_{1}=e_{2}-e_{1}-k u_{1} \\
\dot{e}_{2}=x_{1} z_{1}+c-k x_{2} z_{2}-k c-k u_{2} \\
\dot{e}_{3}=\mu-\exp \left(x_{1} y_{1}\right)-k \mu+k \exp \left(x_{2} y_{2}\right)-k u_{3}
\end{array}\right.
$$

Theorem 1: If the nonlinear feedback synchronization controller is

$$
\left\{\begin{array}{l}
u_{1}=0 \\
u_{2}=\beta_{1}\left(y_{1} / k-y_{2}\right)+x_{1} z_{1} / k+(1 / k-1) c-x_{2} z_{2} \\
u_{3}=(1 / k-1) \mu-\exp \left(x_{1} y_{1}\right) / k+\exp \left(x_{2} y_{2}\right)+\beta_{2}\left(z_{1} / k-z_{2}\right)
\end{array}\right.
$$




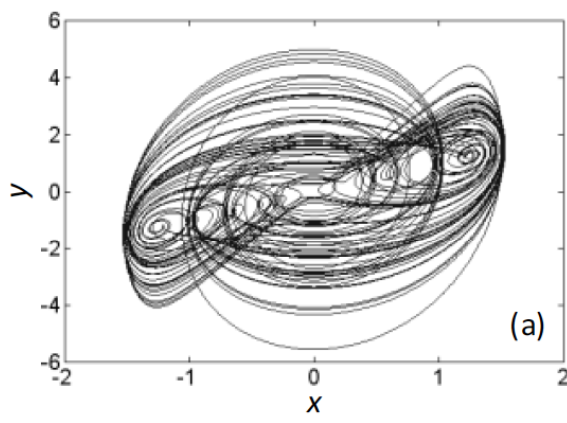

(a) $x-y$ plane projection $(c=0)$

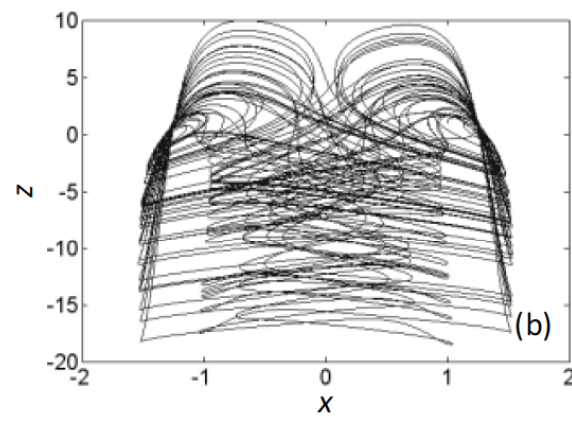

(b) $x-z$ plane projection $(c=0)$

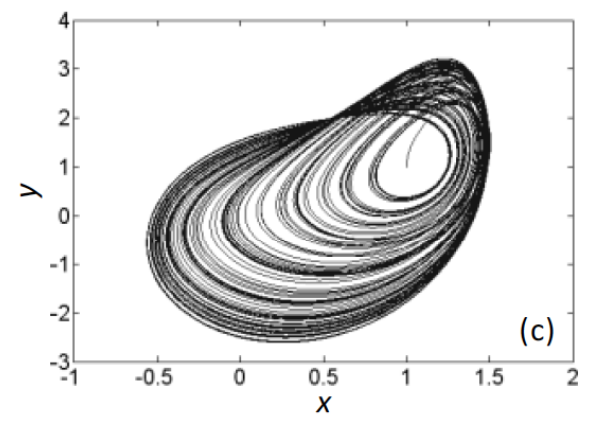

(c) $x-y$ plane projection $(c=2)$

Fig. (1). New chaotic attractors.

Where $\beta_{1}>0$ and $\beta_{2}>0$, then the chaotic systems (2) and (3) with different initial value, can achieve generalized projective synchronization.

Proof: according to the idea of active control, the control functions are designed as follows:

$$
\left\{\begin{array} { l } 
{ \dot { e } _ { 1 } = e _ { 2 } - e _ { 1 } + v _ { 1 } } \\
{ \dot { e } _ { 2 } = v _ { 2 } } \\
{ \dot { e } _ { 3 } = v _ { 3 } }
\end{array} \left\{\begin{array}{l}
k u_{1}=-v_{1} \\
k u_{2}=-v_{2}+x_{1} z_{1}+c-k x_{2} z_{2}-k c \\
k u_{3}=-v_{3}+\mu-\exp \left(x_{1} y_{1}\right)-k \mu+k \exp \left(x_{2} y_{2}\right)
\end{array}\right.\right.
$$

By substituting it into the error system (4) is acquired:

$\left\{\begin{array}{l}\dot{e}_{1}=e_{2}-e_{1}+v_{1} \\ \dot{e}_{2}=v_{2} \\ \dot{e}_{3}=v_{3}\end{array}\right.$

$v_{1}, v_{2}, v_{3}$ are error variables, $e_{1}, e_{2}, e_{3}$ are input control signals of the function. Set up

$\left[\begin{array}{l}v_{1} \\ v_{2} \\ v_{3}\end{array}\right]=A\left[\begin{array}{l}e_{1} \\ e_{2} \\ e_{3}\end{array}\right]=\left[\begin{array}{ccc}0 & 0 & 0 \\ 0 & -\beta_{1} & 0 \\ 0 & 0 & -\beta_{2}\end{array}\right]\left[\begin{array}{l}e_{1} \\ e_{2} \\ e_{3}\end{array}\right]$

By substituting into equation (7), is acquired:

$\left\{\begin{array}{l}\dot{e}_{1}=e_{2}-e_{1} \\ \dot{e}_{2}=-\beta_{1} e_{2} \\ \dot{e}_{3}=-\beta_{2} e_{3}\end{array}\right.$ (9) is:

The corresponding characteristic equation of error system

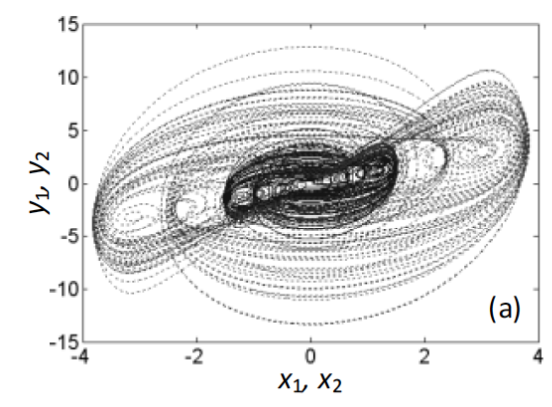

(a) In-phase curve

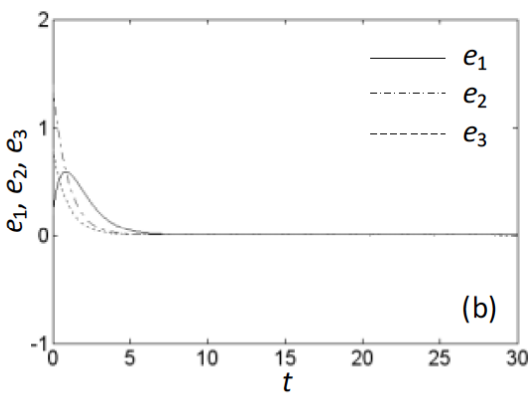

(b) error curve $(\lambda+1)\left(\lambda+\beta_{1}\right)\left(\lambda+\beta_{2}\right)=0$

According to the linear system stability theory, as long as $\beta_{1}>0$ and $\beta_{2}>0$, all characteristic roots of the error system (10) will be negative, so that the error signals $e_{1}, e_{2}, e_{3}$ converge into zero, which means that the zero equilibrium point for the error system is asymptomatically stable.

$\lim _{t \rightarrow \infty} E(t)=\lim _{t \rightarrow \infty}[X(t)-k Y(t)]=0$ shows that two threedimensional chaotic systems obtain same structural generalized projective synchronization with arbitrary proportion. Formula (5) gives the designed controllers that are obtained by substituting formula (8) into (6).

The feature values of error system (10) are related to the speed that error tends to be zero. According to the system stability theory, the smaller the negative eigenvalues, the faster the response of the system. Therefore, from the characteristic equation (10), it is shown that the convergence speed of the error signals is related to the choice of $\beta_{1}$ and $\beta_{2}$. $\beta_{1}$ and $\beta_{2}$ are error convergence speed regulators.

\section{NUMERICAL SIMULATION OF GENERALIZED PROJECTIVE SYNCHRONIZATION}

Using Runge-Kutta method for numerical simulation, a group of parameters $\mu=5, \beta_{1}=1$ were selected, $\beta_{2}=1$, so that the system is in chaotic state, initial values $(1,1,1)$ and $(2,-1,0.5)$ were set for drive system and response system, respectively. And $c=0$, when the scale factors for generalized projective synchronization $k$ are 0.4 and -1.2 respectively, the generalized projective synchronization simulation results are shown in Figs. $(\mathbf{2}, \mathbf{3})$. When the control parameter $c$ is 2 and $k$ is -0.5 , generalized projective synchronization

Fig. (2). Generalized projective synchronization at $\mathrm{K}=0.4$.

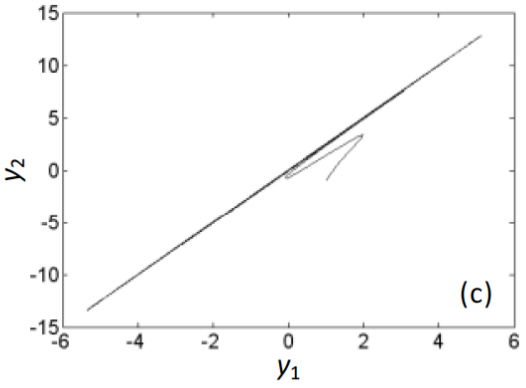

(c) synchronous curve 


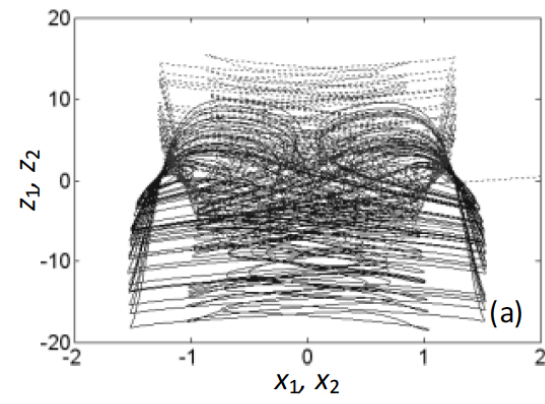

(a) Anti-phase curve

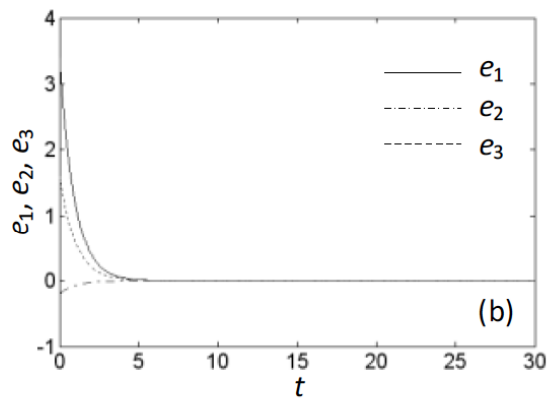

(b) error curve

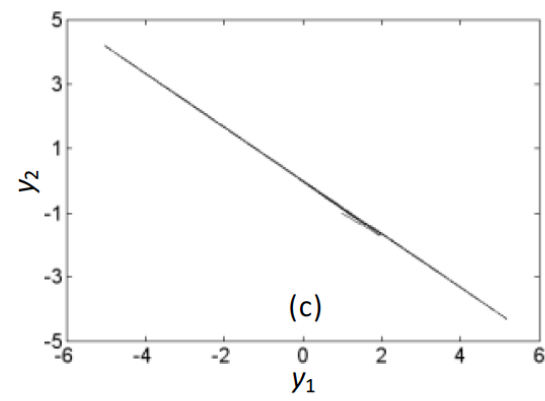

(c) synchronous curve

Fig. (3). Generalized projective synchronization at $\mathrm{K}=-1.2$.

simulation results are shown in Fig. (4). In the picture, the projection phase orbit with solid and dashed lines are attractors of drive system and response system respectively.

Generalized projective synchronization of in-phase as shown in Fig. (2a) indicates the characteristics of the drive system and the response system. Fig. (2b) gives error signal evolution curve at $k=0.4$, error initial values are $e_{1}(0)=0.2, e_{2}(0)=1.4, e_{3}(0)=0.8$, we can see the error curve quickly decays to 0 , indicating that state variables between two three-dimensional chaotic systems are controlled by the circuit.

\section{CIRCUIT DESIGN AND SIMULATION}

We realized generalized projective synchronization of robust chaotic systems with exponential item using the cir- cuit designed in the literature [2]. The control circuit corresponding to the formula (5) is shown in Fig. (5), which achieved state variables in-phase or anti-phase generalized projective synchronization between the drive system and the response system respectively.

The control circuit method shows that in order to realize two systems generalized projective synchronization, it required only to adjust the linear resistor vector $[\mathrm{R} 1 \mathrm{c}, \mathrm{R} 2 \mathrm{c}$, $\mathrm{R} 3 \mathrm{c}, \mathrm{R} 4 \mathrm{c}, \mathrm{R} 5 \mathrm{c}$ ] in proportional action, and the corresponding feedback signal vector [S1c, S2c, S3c, S4c, S5c] is coupled into the response system. The feedback signals, in-phase and anti-phase signals, in the controller are derived from state variables and intermediate variables of drive system and response system respectively. With resistance vector in the controller, the feedback signal is in-

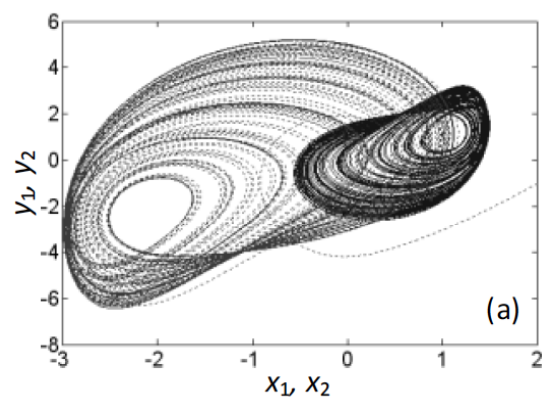

(a) $x-y$ plane projection

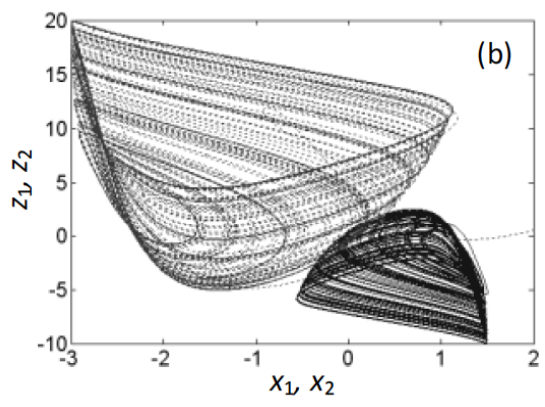

(b) $x-z$ plane projection

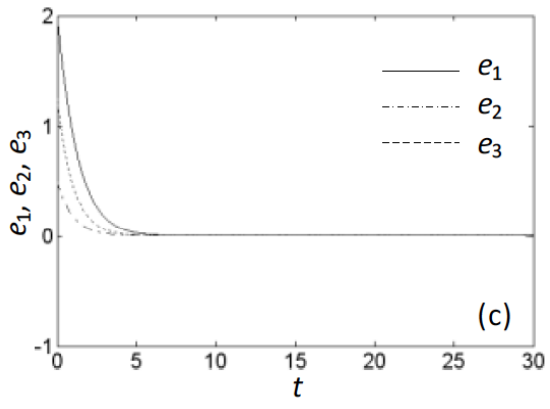

(c) error curve

Fig. (4). Anti-phase synchronization at $\mathrm{c}=2$.

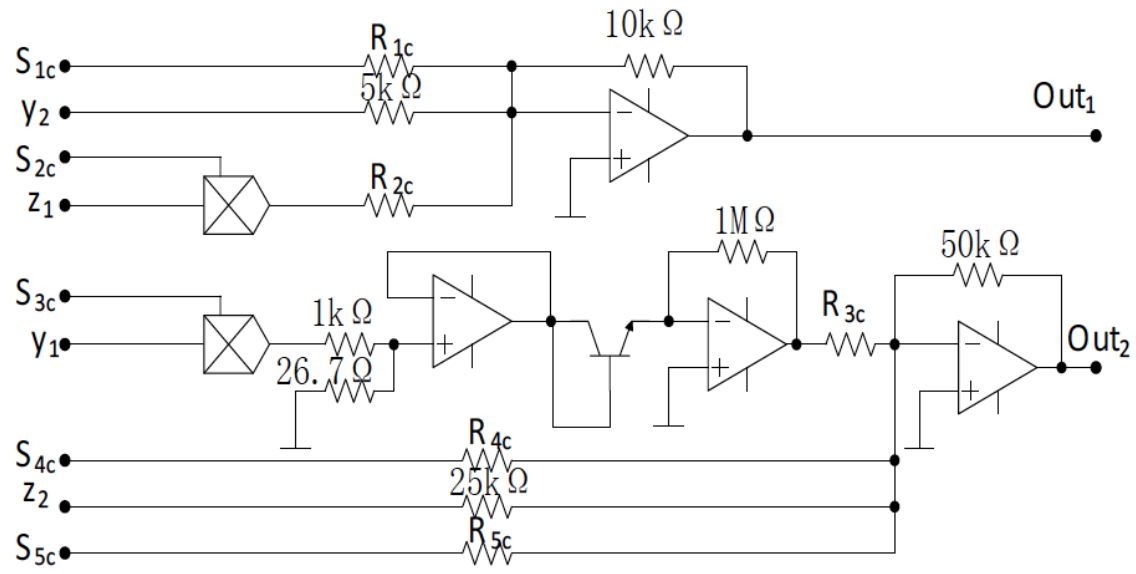

Fig. (5). Example of a ONE-COLUMN figure caption. 


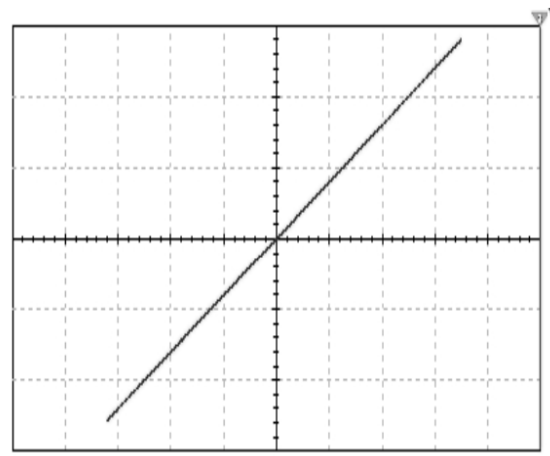

(a) $y_{1}-y_{2}(k=0.4), 1 / 2$

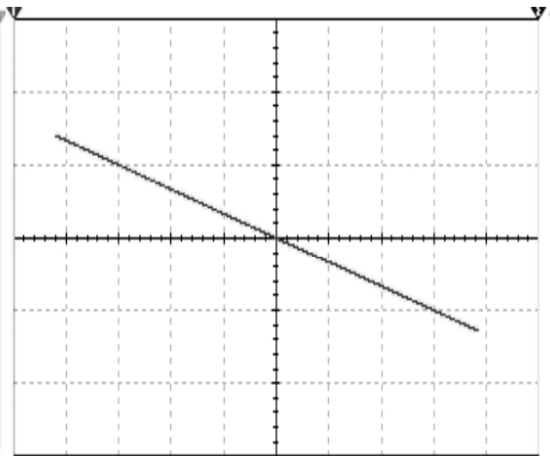

(b) $y_{1}-y_{2}(k=-1.2), 2 / 5$

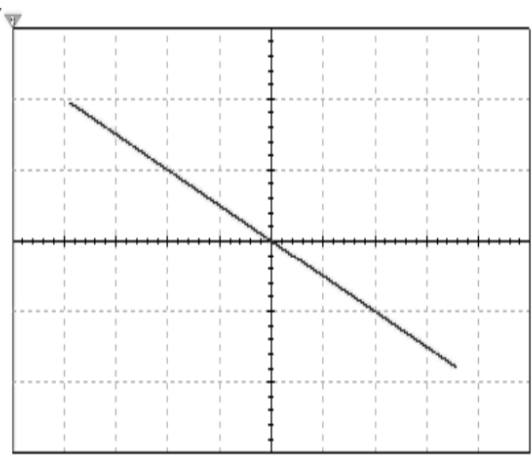

(c) $y_{1}-y_{2}(k=-0.5), 2 / 2$

Fig. (6). Synchronization curves of simulation circuit.

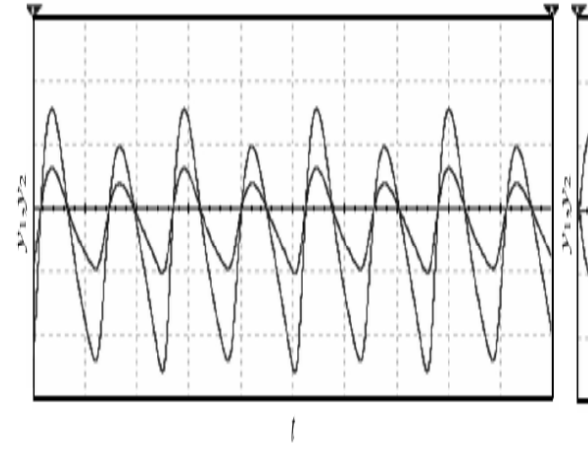

(a) $k=0.4(c=0)$

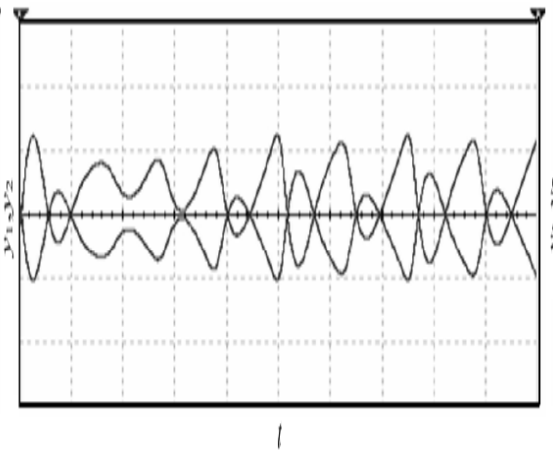

(b) $k=-1.2(c=0)$

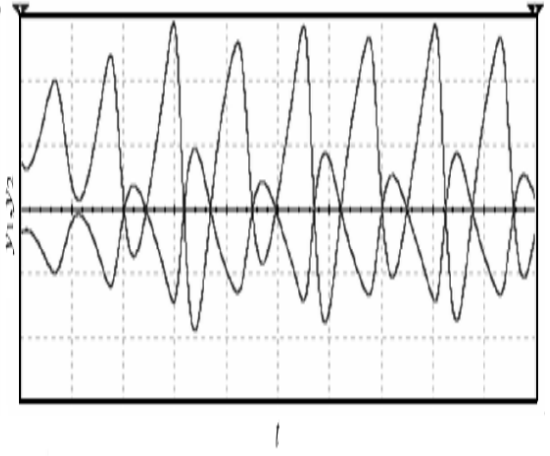

(c) $k=-0.5(c=2)$

Fig. (7). State output signals of the drive system and the response system.

corporated into the addition operation module of the response system as an added signal. In Fig. (5), at $k=0.4$, the feedback signal vectors $\left[S_{1 \mathrm{c}}, S_{2 \mathrm{c}}, S_{3 \mathrm{c}}, S_{4 \mathrm{c}}, S_{5 \mathrm{c}}\right]$ and the controller resistance vectors $\left[R_{1 \mathrm{c}}, R_{2 \mathrm{c}}, R_{3 \mathrm{c}}, R_{4 \mathrm{c}}, R_{5 \mathrm{c}}\right]$ are [$\left.y_{1},-x_{1}, x_{1},-z_{1},-v_{\mathrm{c}}\right]$ and $[2 k \Omega, 4 k \Omega, 20 k \Omega, 10 k \Omega, 4 k \Omega]$, respectively. At $k=-1.2$, the feedback signal vectors $\left[S_{1 \mathrm{c}}\right.$, $\left.S_{2 \mathrm{c}}, S_{3 \mathrm{c}}, S_{4 \mathrm{c}}, S_{5 \mathrm{c}}\right]$ and the controller resistance vectors $\left[R_{1 \mathrm{c}}\right.$, $\left.R_{2 \mathrm{c}}, R_{3 \mathrm{c}}, R_{4 \mathrm{c}}, R_{5 \mathrm{c}}\right]$ are $\left[y_{1}, x_{1}, x_{1}, z_{1}, v_{\mathrm{c}}\right]$ and $[12 \mathrm{k} \Omega, 15 \mathrm{k} \Omega$, $60 \mathrm{k} \Omega, 60 \mathrm{k} \Omega, 50 \mathrm{k} \Omega$ ], respectively.

Circuit simulation was performed according to system parameter values in the fourth part of numerical emulation, the synchronization status between drive system and response system is shown in figure 6. In the graph, $y_{1}-y_{2}$ is the phase trajectory map, coordinate scale unit is $\mathrm{V} / \mathrm{div}$, such as $1 / 2$ means that transverse coordinate of each grid is $1 \mathrm{~V}$, ordinate per cell is $2 \mathrm{~V}$. In Figs. (6a, $\mathbf{b}$ and $\mathbf{c})$, numerical values can be obtained by oscilloscope, the ratio of state variables $y_{1}$ and $y_{2}$ is between $0.4,-1.2,-0.5$, which illustrates the response system and drive system achieved a certain proportion of the generalized projective synchronization.

As shown in Fig. (7), the nonlinear controller designed in same structure generalized projective synchronization can connect the drive system and the response system, through proportional adjustment of the linear resistor vector, and the corresponding feedback signal vector coupled to the response system, so that the two chaotic attractors phase can be same or opposite. The output amplitude when the scale factor is $0.4,-1.2,-0.5(c=2)$, verifies the possibility to achieve generalized projective synchronization between the drive system and the response system with the scale factor.

\section{CONCLUSION}

Analysis of the structural characteristics of chaotic attractor, the generalized projective synchronization controller, and the generalized projective synchronization of chaotic attractor can be verified by numerical simulation and circuit simulation.

\section{CONFLICT OF INTEREST}

The authors confirm that this article content has no conflicts of interest.

\section{ACKNOWLEDGEMENT}

This work is supported by Changzhou New Technology Laboratory of Internet of Things, China (No.CM20133008).

\section{REFERENCES}

[1] B. C. Bao, C. B. Li, J. P. Xu, and Z. Liu, "New robust chaotic system with exponential quadratic term," Chinese Phys., vol. 17, no.11, pp. 4022-4026, 2008.

[2] B. C. Bao, X. Y. Mao, Z. Liu, "Circuit realization for a new threedimensional chaotic system with exponential function," The 21st circuits and Systems Conference, pp. 35-39, 2008. 
[3] J. Liu, and J. A. Lu, "Projective synchronization in a circulant partially linear chaotic system and its control," Int. J Information Technology, vol. 12, no.5, pp. 125-134, 2006.

[4] J. Yan, and C. Li, "Generalized projective synchronization of a unified chaotic system," Chaos, Solitons and Fractals, vol. 26, no.4, pp. 1119-1124, 2005.
[5] C. Li, and J. Yan, "Generalized projective synchronization of chaos: The cascade synchronization approach," Chaos, Solitons and Fractals, vol. 30, no.1, pp. 140-146, 2006.

Received: September 16, 2014

Revised: December 23, 2014

Accepted: December 31, 2014

(C) Bi et al.; Licensee Bentham Open.

This is an open access article licensed under the terms of the (https://creativecommons.org/licenses/by/4.0/legalcode), which permits unrestricted, non-commercial use, distribution and reproduction in any medium, provided the work is properly cited. 